\title{
The Association between Physical Activity and Psychological Needs in College-Aged Mexican-American Males
}

\author{
VANESSA MARTINEZ, CHENG-CHEN PAN, ROBERT REGUENES, GABRIELA \\ ALLEN, CHARITY CAVAZOS, DAVID WITTENBURG, \& JARED MONTOYA
}

The University of Texas at Brownsville

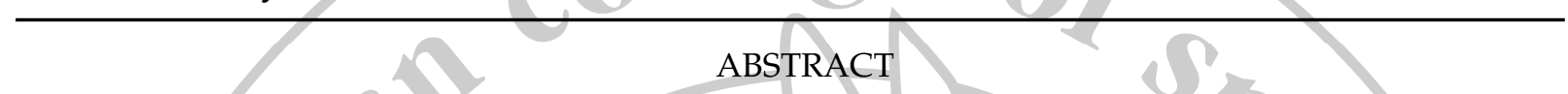

In the last several decades, there has been a tremendous wave of interest in the overweight/obese population in the United States. However, comparatively little research has focused on the association between psychological needs and physical activity (PA) of college-aged Mexican-American males. PURPOSE: To investigate the relationships between PA, obesity, and psychological needs for exercise among college-aged Mexican-American males. METHODS: Height, weight, waist circumference (WC), and BMI measures were taken on 207 Mexican-American college-aged students $(\mathrm{M}=22.59 \pm 5.37)$. PA was assessed using The International Physical Activity Questionnaire (IPAQ) to determine light, moderate, or vigorous PA level per week. Need Satisfaction was assessed utilizing the Psychological Need Satisfaction in Exercise Scale (PNSE). The PNSE examines degrees of Perceived Competence (PC), Perceived Autonomy (PAu), and Perceived Relatedness (PR) as they pertain to an individual's motivation to exercise. RESULTS: The results indicate that $65.7 \%$ subjects are overweight or obese according to BMI standards. There were significant correlations between moderate (3-5 days or 600 MET-minutes/wk) and vigorous ( $\geq 3$ days or 1500 MET-minutes/wk) PA levels, BMI, and WC. Significant relationships between BMI, PC, and PAu were also observed. WC was significantly related to PC. Additionally, moderate and vigorous levels of PA are positively correlated with PC $(\mathrm{r}=.311, .366, \mathrm{p}<.05), \mathrm{PAu}(\mathrm{r}=.153, .239, \mathrm{p}<.05)$ and PR $(\mathrm{r}=171,248, \mathrm{p}<.05)$. Walking was not associated with healthy BMI and WC. CONCLUSION: Findings indicate Mexican-American males who reported greater levels of moderate and vigorous PA had reduced BMI and WC, except walking. Participants engaged in walking with low levels of moderate PA had unhealthy BMI and WC. Mexican-American males who engage in high levels of moderate and vigorous PA are more likely to have higher perceived PNSE scores and reduced BMI and WC. Increased emphasis should be on intrinsic motivation for individuals to adopt healthy lifestyles that adhere to at least 3 days of vigorous PA per week. Therefore, successful intervention programs need to focus on pairing the psychological needs for exercise with the actual exercise program.

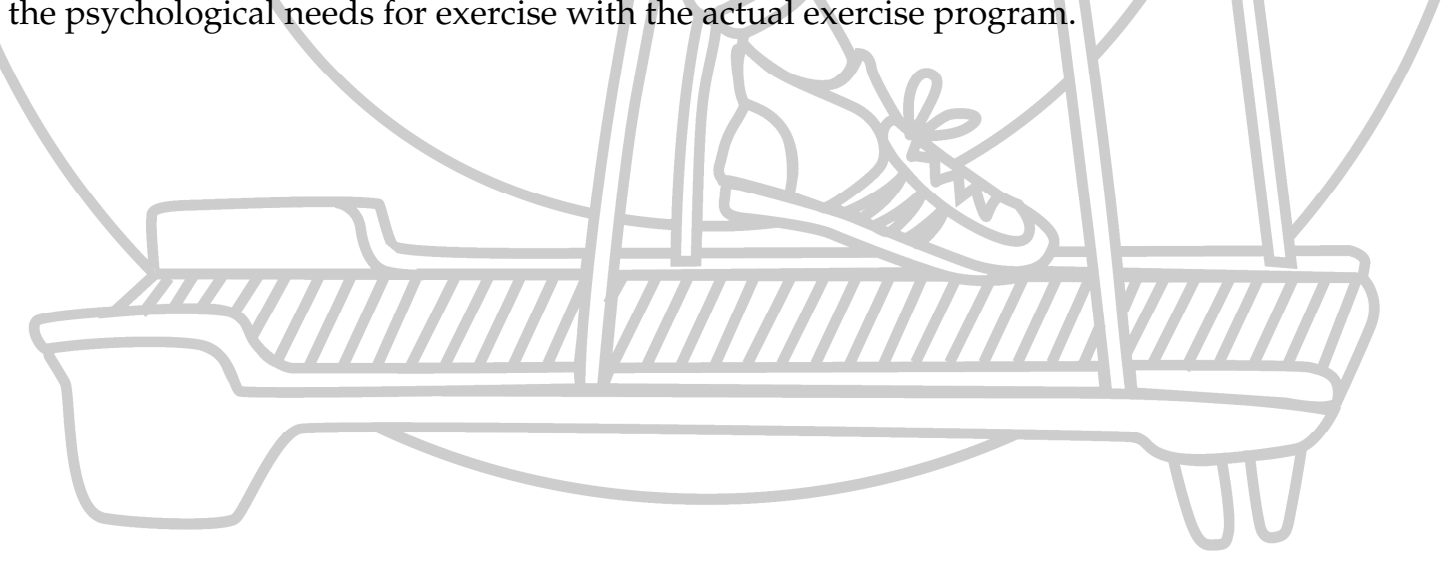

